EPJ Web of Conferences 82, 01058 (2015)

DOI: $10.1051 /$ epjconf/20158201058

(C) Owned by the authors, published by EDP Sciences, 2015

\title{
Experimental study of evaporation of horizontal films of water-salt solutions
}

\author{
S.L. Elistratov and V.S. Morozov \\ Kutateladze Institute of Thermophysics SB RAS, Novosibirsk, Russia
}

\begin{abstract}
The present studies were carried out for the horizontal films (thin layers) of water and water solutions of $\mathrm{NaCl}, \mathrm{CaCl}_{2}, \mathrm{LiCl}$, and $\mathrm{LiBr}$ with different solubility characteristics, as well as with specific features of formation and decay of water hydrates. Required volume of solution $V_{o}$ of given weight concentration $\xi_{o}$, preliminary heated to the working surface temperature, was put in one step on the horizontal bottom of the bowl, heated to working temperature $t_{C T}$, by means of volume batchers Thermo Scientific. After evaporation completion, the final mass of solution and form of their residue were registered. At the final stage of evaporation formation of $\mathrm{NaCl}$ crystals and water hydrates of $\mathrm{CaCl}_{2} \cdot 2 \mathrm{H}_{2} \mathrm{O}, \mathrm{LiCl} \cdot \mathrm{H}_{2} \mathrm{O}$, and $\mathrm{LiBr} \cdot 2 \mathrm{H}_{2} \mathrm{O}$ occurred.
\end{abstract}

The processes of evaporation and boiling of liquid films are one of important directions of development of modern thermal physics. This problem becomes much more complicated, when the objects of study are different kinds of mixtures and solutions used in refrigeration, power engineering, petrochemistry and chemical technologies. Evaporation of solutions is accompanied by changes in their concentration; under the certain conditions separation of the solid phase (crystals of salt hydrates) is also possible. The present studies were carried out for the horizontal films (thin layers) of water and water solutions of $\mathrm{NaCl}, \mathrm{CaCl}_{2}, \mathrm{LiCl}$, and $\mathrm{LiBr}$ with different solubility characteristics, as well as with specific features of formation and decay of water hydrates.

The scheme of experimental setup is shown in Fig. 1. The working section of this setup was located on the analytical balance AJ-6200CE with resolution of $0.01 \mathrm{~g}$ at maximal measuring mass of $6200 \mathrm{~g}$. The use of special flexible conducting connections allowed us to neutralize the effect of thermocouple and electric power wires on accuracy and dynamics of weighting. On the inner surface of the bowl, contacting with solutions, there was an anticorrosive thin hydrophobic ceramic coating. In preliminary experiments, we determined emissivity $\varepsilon$ of ceramic coating, dynamic response of balance; adjusted the method for maintaining a horizontal heating surface; and stabilize the temperature of the working surface with the accuracy $\pm 1.0^{\circ} \mathrm{C}$. Weight concentration of solutions $\xi$ was determined by tabular data on the basis of readings of the standard densimeters adjusted for the solution temperature.

Required volume of solution $V_{o}$ of given weight concentration $\xi_{o}$, preliminary heated to the working surface temperature, was put in one step on the horizontal bottom of the bowl, heated to working temperature $t_{C T}$, by means of volume batchers Thermo Scientific. To visualize evaporation of the horizontal layers of solution, the digital camera and thermal imager, whose readings were synchronized with weight measurements, were used. The temperature of the surface of evaporating liquid films

This is an Open Access article distributed under the terms of the Creative Commons Attribution License 4.0, which permits unrestricted use, distribution, and reproduction in any medium, provided the original work is properly cited. 


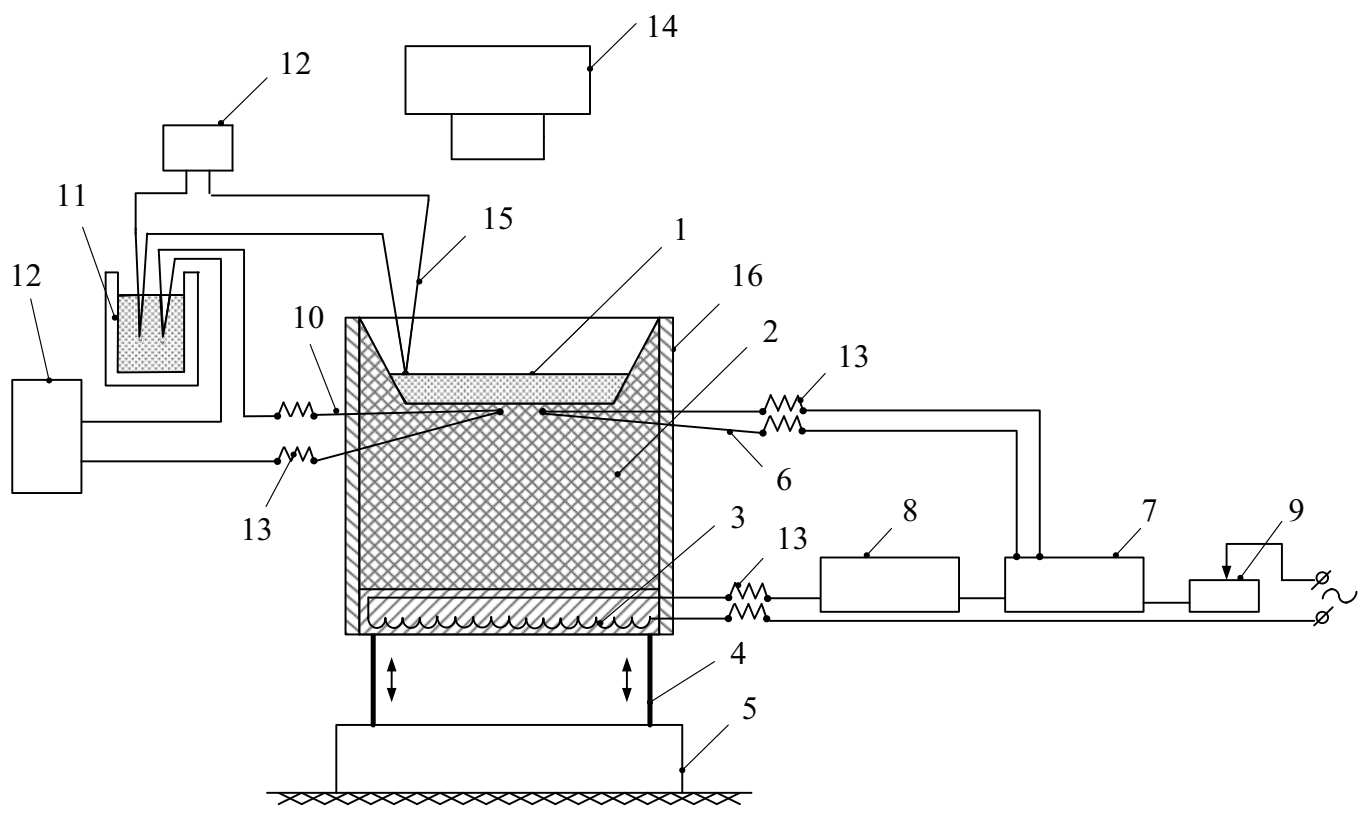

Figure 1. The scheme of setup. 1 - films (layers) of water-salt solutions; 2 - aluminum alloy bowl with a thick bottom; 3 - regulated electric heater; 4 - adjustable supports; 5 - analytical balance; 6 - thermocouple for heating regulation; 7 - regulator-temperature meter OVEN; 8 - electrical relay; 9 - regulated electric transformer; 10 - individually calibrated thermocouple; 11 - flask with melting ice; 12 - multimeter Aktakom; 13 - flexible conducting connections; 14 - thermal imager TermoTracer TH 7102 with wavelength $10.6 \mu$ m, camera Panasonic DMC-TZ2, pyrometer Aktakom ATE - 256; 15 - measuring copper-constantan thermocouple; 16 - thermal insulation.

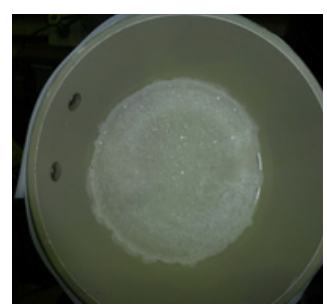

$\mathrm{NaCl}$

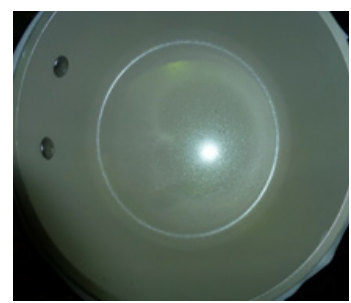

$\mathrm{CaCl}_{2}$

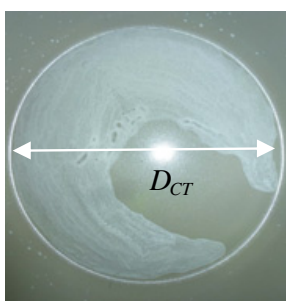

$\mathrm{LiCl}$

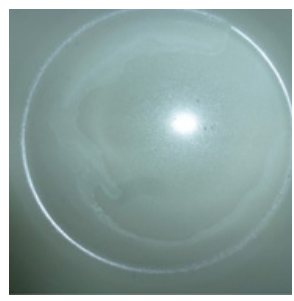

$\mathrm{LiBr}$

Figure 2. General types of evaporation residue of solid horizontal films of water-salt solutions with initial volume $V_{o}=5 \cdot 10^{-5} \mathrm{~m}^{3}$ at $t_{C T}=80^{\circ} \mathrm{C}$.

were also measured during evaporation by thermocouple 10, calibrated individually. After evaporation completion, the final mass of solution and form of their residue were registered (see Fig. 2).

At the final stage of evaporation (see Fig. 2) formation of $\mathrm{NaCl}$ crystals and water hydrates of $\mathrm{CaCl}_{2} \cdot 2 \mathrm{H}_{2} \mathrm{O}, \mathrm{LiCl} \cdot \mathrm{H}_{2} \mathrm{O}$, and $\mathrm{LiBr} \cdot 2 \mathrm{H}_{2} \mathrm{O}$ occurred. Formation and thermal decomposition of salt hydrates depending on the surface temperature is one of the main features of studying physics of evaporation of the thin films of water-salt solutions.

In Fig. 3 the vertical lines indicate time, when a round shape was distorted under the influence of the surface tension. The initial thickness of the layer in our experiments at working surface diameter $D_{C T}=$ $1.43 \cdot 10^{-2} \mathrm{~m}$ was $\delta_{0} \approx 3.1 \mathrm{~mm}$, and distortions of a round shape of films occurred at $\delta_{0} \leq 0.36 \mathrm{~mm}$. 


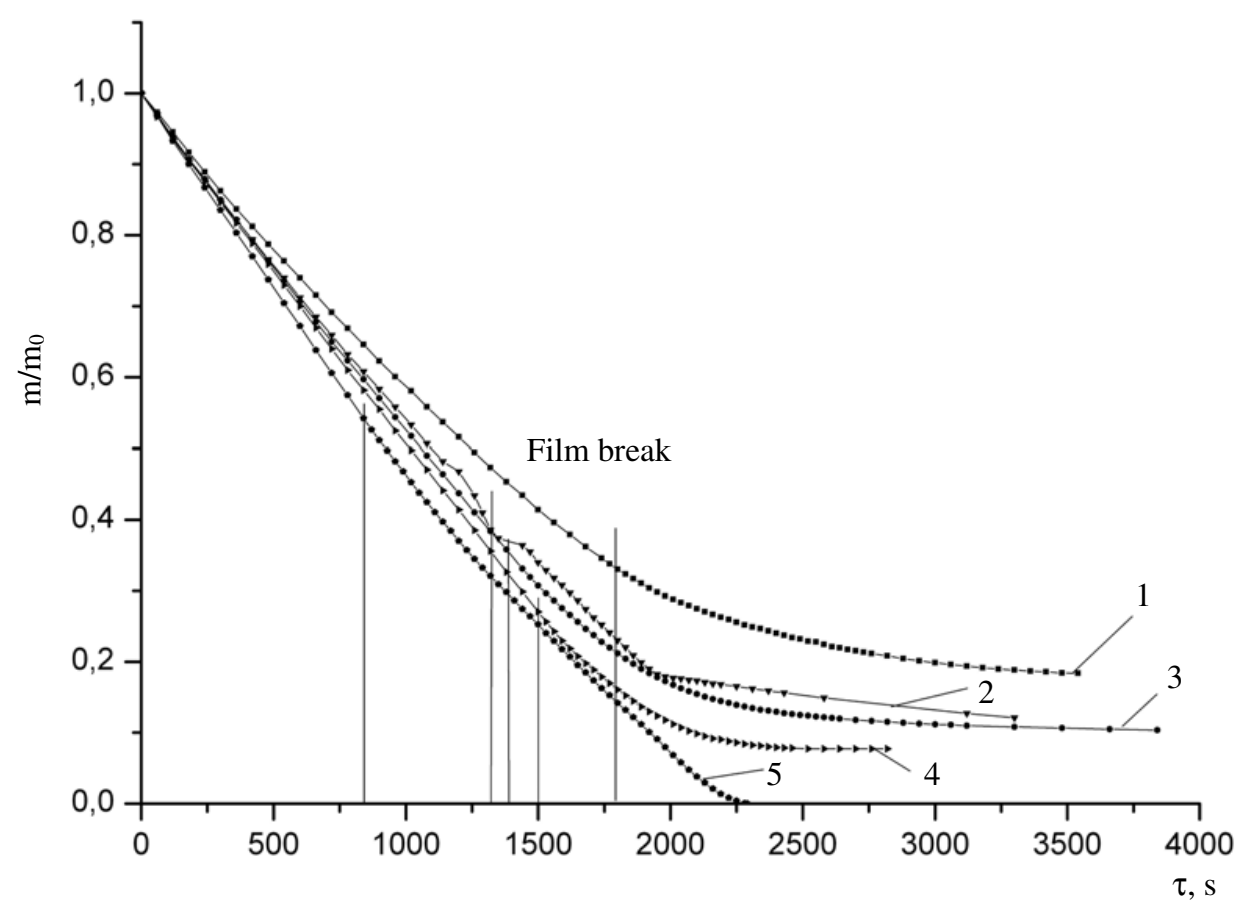

Figure 3. Comparison of relative rates of evaporation of horizontal films (layers) of water-salt solutions with initial volume $V_{o}=5 \cdot 10^{-5} \mathrm{~m}^{3}$ and weight concentration $\xi_{0}=10.0 \%$ at $t_{C T}=80{ }^{\circ} \mathrm{C}$. 1 - water $+\mathrm{LiCl} ; 2-$ water $+\mathrm{NaCl} ; 3-$ water $+\mathrm{LiBr} ; 4-$ water $+\mathrm{CaCl}_{2} ;-$ distilled water.

Based on dependences of a mass change (see Fig. 3) we can determine the average heat transfer coefficient at film evaporation until the beginning of distortion of their round shape:

$$
\alpha=\frac{4 \cdot \Delta m \cdot R}{\Delta \tau \cdot \pi \cdot D_{C T}^{2}\left(t_{C T}-t_{O C}\right)},
$$

where $\Delta m$ is the mass of water evaporated from the film, $\mathrm{kg} ; R$ is latent heat of water evaporation, $\mathrm{kJ} / \mathrm{kg}$; $\Delta \tau$ is time of evaporation, $\mathrm{s} ; t_{O C}$ is ambient temperature in the room, ${ }^{\circ} \mathrm{C}$.

In our experiments, the heat transfer coefficients were $58,46,59,57 \mathrm{~W} / \mathrm{m}^{2} \cdot{ }^{\circ} \mathrm{C}$ for water solutions of $\mathrm{LiBr}, \mathrm{LiCl}, \mathrm{CaCl}_{2}, \mathrm{NaCl}$ and $63 \mathrm{~W} / \mathrm{m}^{2} \cdot{ }^{\circ} \mathrm{C}$ for water. A decrease in heat transfer intensity for water solutions of salts is caused by depression of pressure of saturated water vapors above their surface [1].

Experimental results allowed the conclusion about reasonability of suggested methods for studying the physics of transfer processes during evaporation, boiling, condensation, non-isothermal absorption (adsorption) and desorption of thin liquid and solid horizontal films of complex composition for a wide range of temperatures of the heating surface.

The work was supported by RSF grant No. 14-19-00352.

\section{Reference}

[1] V.N. Filatkin. Thermodynamics of solutions: tutorial.- St.Petersburg: State University of LowTemperature and Food Technologies, 187 (2000) 